\title{
Adherencia declarada y calculada al consumo de chocolate en los participantes de un ensayo clínico que mide el efecto del cacao en la hipertensión arterial con diagnóstico reciente. Medellín, Colombia
}

\author{
María Camila Cardona-0campo ${ }^{1}$, Mónica Lucía Giraldo-Restrepo ${ }^{2}$, Luz Ángela Angarita-Fuentes³ \\ Carlos José Jaramillo-Gómez ${ }^{4}$, Pablo Javier Patiño-Grajales ${ }^{5}$, Héctor Iván García-García ${ }^{6}$
}

La OMS define la adherencia a un tratamiento como "la medida en que la conducta de una persona corresponde con las recomendaciones acordadas con un proveedor de atención médica" (1). Si este concepto se aplica al control de la hipertensión arterial (HTA), incluye cambios duraderos en el estilo de vida que, algunas veces, se acompañan del uso de medicamentos. La primera línea de atención y manejo de la HTA son las medidas no farmacológicas que cuentan con el respaldo de las guías internacionales de mayor difusión como son el Joint National Committee on Prevention, Detection, Evaluation and Treatment of High Blood Pressure (JNC-VIII) ${ }^{(2)}$ y European Society of Hypertension-European Society of Cardiology Guidelines (ESH-ESC) ${ }^{(3)}$.

Las recomendaciones incluyen cambios en el estilo de vida relacionados con la alimentación, la actividad física, el consumo de sodio, alcohol y tabaco; además, se ha estudiado el efecto potencialmente benéfico de algunos alimentos en la reducción del riesgo cardiovascular, entre ellos el cacao, que por su alto contenido de polifenoles, actuaría como antioxidante en el tejido endotelial ${ }^{(4)}$.

Algunos trabajos indican que el consumo de cacao puede disminuir la presión arterial, aproximadamente $2 \mathrm{mmHg}$ en personas hipertensas ${ }^{(4)}$. En estos pacientes se encuentra que la

1 Estudiante de Medicina, Joven Investigadora, Grupo Académico de Epidemiología Clínica-GRAEPIC, Universidad de Antioquia, Medellín, Colombia.

2 Enfermera. MSc en Ciencias Básicas Biomédicas. Sc.D. Bioquímica e Inmunología. Profesora Facultad de Medicina, Grupo de Inmunodeficiencias Primarias, Universidad de Antioquia, Medellín, Colombia.

3 Médica y Cirujana, MSc en Ciencias Clínicas, Grupo Académico de Epidemiología Clínica-GRAEPIC, Universidad de Antioquia, Medellín, Colombia.

4 Médico y Cirujano. Especialista en Medicina Interna y Cardiología Clínica, Profesor Facultad de Medicina, Universidad de Antioquia, Medellín, Colombia.

5 Médico y Cirujano, MSc en Inmunología. Sc.D. Ciencias Básicas Biomédicas. Profesor Facultad de Medicina. Grupo Inmunodeficiencias Primarias, Universidad de Antioquia, Medellín, Colombia.

6 Médico y Cirujano, MSc en Salud Pública, MSc en Epidemiología. Profesor Facultad de Medicina, Grupo Académico de Epidemiología Clínica-GRAEPIC, Universidad de Antioquia, Medellín, Colombia.

Correspondencia: Mónica Lucía Giraldo-Restrepo; monica.giraldo1@udea.edu.co

Recibido: enero 4 de 2018

Aceptado: noviembre 22 de 2018

Cómo citar: Cardona-Ocampo MC, Giraldo-Restrepo ML, Angarita-Fuentes LA, Jaramillo-Gómez CJ, Patiño-Grajales PJ, García-García HI . Adherencia declarada y calculada al consumo de chocolate en los participantes de un ensayo clínico que mide el efecto del cacao en la hipertensión arterial con diagnóstico reciente. Medellín, Colombia. Iatreia. 2019 Oct-Dic;32(4):354-357. DOI. 10.17533/ udea.iatreia.17. 
adherencia a cada una de las medidas no farmacológicas es variable ${ }^{(5,6)}$, su reducción limita sus beneficios y, en última instancia, conlleva al deterioro paulatino de la salud. Las explicaciones para esta disminución incluyen problemas en la prescripción, fallas en la interacción entre el médico y el paciente, así como la falta de motivación, participación y conocimiento del paciente con respecto a la enfermedad y su tratamiento.

El objetivo del presente estudio fue comparar la adherencia, por informe real y reportado por los pacientes, al consumo de cacao como medida no farmacológica en una población adulta económicamente activa, con un diagnóstico reciente de HTA que participó en un ensayo clínico controlado sobre el efecto del consumo de cacao.

\section{MÉTODOS}

Se realizó un análisis secundario con los datos obtenidos de los sujetos en la investigación Ensayo clínico controlado del efecto del consumo de cacao en parámetros cardiovasculares de pacientes con diagnóstico reciente de hipertensión arterial esencial inscritos en un programa de manejo no farmacológico en una EPS de Antioquia (ClinicalTrials.gov: NCT01496235), que se ejecutó entre febrero de 2009 y julio de 2011.

Los participantes se asignaron aleatoriamente para recibir diariamente y durante 12 semanas, barras de chocolate de 50 g con $70 \%$ de sólidos de cacao $(\mathrm{n}=34)$, el cual se denominó cacao negro, o para recibir barras de chocolate exentas de sólidos de cacao $(n=32)$, las cuales se denominaron chocolate blanco. Se realizó la evaluación médica y nutricional y, además del chocolate, a ambos grupos se dieron recomendaciones generales sobre medidas no farmacológicas acerca de una alimentación saludable, la realización de actividad física aeróbica, control de la ansiedad y reducción del consumo de bebidas alcohólicas.

La adherencia al tratamiento no farmacológico de la HTA se evaluó con un cuestionario aplicado por una enfermera, diligenciado en cada una de las seis citas de seguimiento realizadas en la Sede de Investigación Universitaria (SIU) de la Universidad de Antioquia (Medellín, Colombia). Se indagó por el cumplimiento de las recomendaciones así como por las dificultades para adherirse a ellas durante el período al que se refería el seguimiento.

Las preguntas versaron sobre el consumo de frutas $y$ vegetales, la realización de actividad física y el control del estrés, así como la reducción del consumo de sal, grasas y bebidas alcohólicas. Además, se verificó que el participante no hubiera consumido tabaco en el período evaluado, es decir cuatro semanas. Para medir la adherencia al consumo de chocolate se utilizó un formato en el que el participante registró su consumo diario de chocolate (adherencia declarada).

Para facilitar el registro diario, el participante señaló en un esquema gráfico de la barra de chocolate, la cantidad que verdaderamente consumió. Este formato también permitió incluir observaciones sobre la palatabilidad y otros efectos atribuibles al chocolate.

Con base en la recolección de los empaques vacíos o las barras de chocolate que el participante devolvió en las visitas de seguimiento, la enfermera calculó el consumo (adherencia calculada). En el ensayo clínico, la recolección de los empaques vacíos, las barras de chocolate devueltas y el auto registro del chocolate consumido, fueron los criterios que se emplearon para establecer la adherencia. Para cada participante se determinó la adherencia declarada y la calculada y se consideró adherente cuando fue mayor o igual al $80 \%$. El acuerdo entre la adherencia declarada y la calculada se evaluó con el Coeficiente kappa y su intervalo de confianza del $95 \%$.

\section{RESULTADOS}

Se incluyeron 66 pacientes de 18 a 65 años de edad, económicamente activos, con un diagnóstico reciente de HTA esencial, sin evidencia de daño orgánico secundario, obesidad, ni consumo de tabaco. Del total de los participantes, 55 (84 \%) fueron adherentes al tratamiento asignado con base en el consumo que declararon y $35(54 \%)$ con base en el cálculo a partir de los empaques devueltos. En el grupo que recibió el chocolate negro, 16 (47\%) participantes tuvieron una adherencia calculada superior al $80 \%$, mientras que en el grupo del chocolate blanco fueron 19 (59\%) personas. La adherencia declarada fue de 28 personas ( $82 \%$ ) del grupo de cacao y 27 (84\%), en el grupo que recibió el chocolate blanco. El Coeficiente kappa entre el reporte 
calculado y el declarado fue 0,21 (IC95 \%: -0,03 a 0,44) para el grupo del chocolate negro y, 0,28 (IC95 \%: -0,01 a 0,58) para el grupo del chocolate blanco. En la Figura 1 se presentan los porcentajes de acuerdo con la adherencia declarada y calculada.

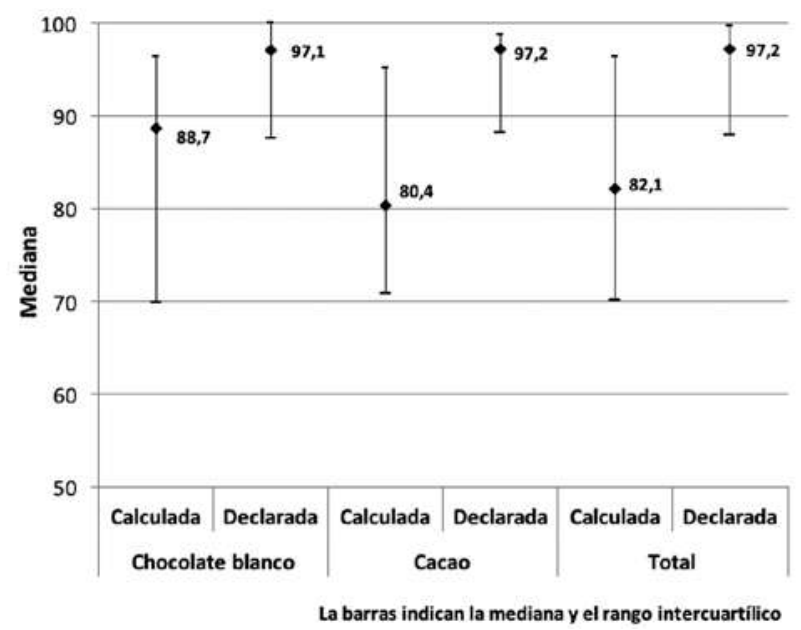

Figura 1. Porcentaje individual de la adherencia calculada y declarada del consumo de cacao (mínimo, mediana y máximo)

\section{DISCUSIÓN}

El diagnóstico y tratamiento integral de las enfermedades crónicas como la HTA implican esfuerzos del personal sanitario y son un reto para la salud pública, porque para mejorar la condición individual no es suficiente la aplicación de las guías de práctica clínica, sino que debe promoverse su comprensión, con el objeto de que el paciente se empodere del autocuidado y se logren las metas terapéuticas ${ }^{(1)}$.

Existen suficientes evidencias sobre los beneficios de las medidas no farmacológicas como primera línea de tratamiento de la HTA ${ }^{(1-3)}$. En general, la adherencia a estas medidas es elevada ${ }^{(5)}$; sin embargo, su aplicación se ve limitada porque los profesionales se marginan de las guías establecidas ya que consideran que los medicamentos antihipertensivos son una opción efectiva que demanda poca inversión en procesos de educación en salud, a diferencia de las terapias no farmacológicas que requieren una mayor inversión de tiempo para la educación y seguimiento. No obstante, esta práctica médica eleva el costo económico para el sistema de salud y expone al paciente a los efectos secundarios de medicamentos cuyo uso debería justificarse con criterios clínicos de claro beneficio y no según la conveniencia para el médico.

En las condiciones del ensayo clínico al que se refiere este análisis, se encontró que la adherencia calculada del consumo de cacao fue menor a las cifras publicadas por otros autores, quienes describen porcentajes cercanos al $90 \%{ }^{(6)}$. En nuestro caso, la adherencia total fue de $54 \%$. Esto podría depender de múltiples variables, entre ellas la palatabilidad del chocolate y los efectos que los participantes pudieron haber atribuido a su consumo (cefalea, dolor abdominal, gastritis). Otros factores que se han descrito para la baja adherencia en los ensayos clínicos, y que también pudieron haber afectado nuestros hallazgos, son la duración prolongada de la intervención y los patrones culturales de consumo de cacao en la alimentación, así como la alta dosis diaria de chocolate (70 gr), entre otros ${ }^{(7)}$.

En ambos grupos del tratamiento la adherencia declarada por los participantes fue superior a la adherencia calculada por la devolución de los empaques vacíos o Ias barras no consumidas $y$, adicionalmente, el acuerdo entre ambos tipos de medidas fue muy pobre.

Una limitación del estudio es el tamaño de la muestra, de manera que las variaciones en los reportes de pocos pacientes pudieron afectar considerablemente el rango de adherencia, lo cual se aprecia claramente en el grupo asionado al chocolate blanco.

Con base en los resultados, la recomendación para incluir el cacao en la alimentación de los pacientes hipertensos como terapia adyuvante en el manejo no farmacológico de su enfermedad debe explorarse más, para establecer el efecto en la reducción de la presión arterial y de los factores de riesgo asociados. No obstante, para optimizar el tratamiento y seguimiento de las enfermedades crónicas no trasmisibles, es necesario que los profesionales acojan las guías de práctica clínica, de tal manera que se haga un uso racional de los medicamentos y se promueva hábitos de vida saludable que incluyan la actividad física regular, el control del estrés y una dieta balanceada y diversa en la que estén los sólidos del cacao, con el fin de reducir el riesgo cardiovascular en la población adulta ${ }^{(3)}$. 


\section{AGRADECIMIENTOS}

Investigación financiada por Colciencias, código 111540820423.

\section{CONFLICTOS DE INTERESES}

Ninguno por declarar.

\section{REFERENCIAS BIBLIOGRÁFICAS}

1. Geest SD, Sabaté E. Adherence to Long-Term Therapies: Evidence for Action. Eur J Cardiovasc Nurs. 2003;2(4):323-323. DOI10.1016/S1474-5151(03)00091-4.

2. James PA, Oparil S, Carter BL, Cushman WC, Dennison-Himmelfarb C, Handler J, et al. Evidence-Based Guideline for the Management of High Blood Pressure in Adults Report From the Panel Members Appointed to the Eighth Joint National Committee (JNC 8). JAMA. 2014;311(5):507-520. DOI 10.1001/jama.2013.284427.

3. Mancia G, Fagard R, Redon J, Narkiewicz K, Zanchetti A, Böhm M, et al. $2013 \mathrm{ESH} / \mathrm{ESC}$ guidelines for the management of arterial hypertension: the Task Force for the Management of Arterial Hypertension of the European Society of Hypertension (ESH) and of the European Society of Cardiology (ESC). Eur Heart J. 2013;34(28):2159-219. DOI 10.1093/eurheartj/eht151.

4. Ried K, Fakler P, Stocks NP. Effect of cocoa on blood pressure. Cochrane Database Syst Rev. 2017;4. DOI: 10.1002/14651858.CD008893.pub3.

5. Xavier D, Gupta R, Kamath D, Sigamani A, Devereaux P, George N, et al. Community health worker-based intervention for adherence to drugs and lifestyle change after acute coronary syndrome: a multicentre, open, randomised controlled trial. Lancet Diabetes Endocrinol. 2016;4(3):244-53. DOI 10.1016/S22138587(15)00480-5.

6. Engler MB, Engler MM, Chen CY, Malloy MJ, Browne A, Chiu EY, et al. Flavonoid-Rich Dark Chocolate Improves Endothelial Function and Increases Plasma Epicatechin Concentrations in Healthy Adults. J Am Coll Nutr. 2004;23(3):197-204. DOI 10.1080/07315724. 2004.10719361.

7. Boudes P. Drug Compliance in Therapeutic Trials: A Review. Control Clin Trials. 1998;19:257-68. 